\title{
Short-Term Precision Error of Body Composition Assessment Methods in Resistance-Trained Male Athletes
}

\author{
Ava Farley and Gary J. Slater \\ Karen Hind \\ University of the Sunshine Coast \\ Durham University
}

\begin{abstract}
Athletic populations require high-precision body composition assessments to identify true change. Least significant change determines technical error via same-day consecutive tests but does not integrate biological variation, which is more relevant for longitudinal monitoring. The aim of this study was to assess biological variation using least significant change measures from body composition methods used on athletes, including surface anthropometry (SA), air displacement plethysmography (BOD POD), dual-energy X-ray absorptiometry (DXA), and bioelectrical impedance spectroscopy (BIS). Thirty-two athletic males (age $=31 \pm 7$ years; stature $=183 \pm 7 \mathrm{~cm}$; mass $=92 \pm 10 \mathrm{~kg}$ ) underwent three testing sessions over 2 days using four methods. Least significant change values were calculated from differences in Day 1 Test 1 versus Day 1 Test 2 (same-day precision), as well as Day 1 Test 1 versus Day 2 (consecutive-day precision). There was high agreement between same-day and consecutiveday fat mass and fat-free mass measurements for all methods. Consecutive-day precision error in comparison with the same-day precision error was $50 \%$ higher for fat mass estimates from BIS (3,607 vs. 2,331 g), 25\% higher from BOD POD (1,943 vs. $1,448 \mathrm{~g}$ ) and DXA (1,615 vs. 1,204 g), but negligible from SA (442 vs. $586 \mathrm{~g})$. Consecutive-day precision error for fat-free mass was $50 \%$ higher from BIS (3,966 vs. 2,276 g) and SA (1,159 vs. $568 \mathrm{~g})$ and $25 \%$ higher from BOD POD (1,894 vs. 1,450 g) and DXA (1,967 vs. $1,461 \mathrm{~g})$ than the same-day precision error. Precision error in consecutive-day analysis considers both technical error and biological variation, enhancing the identification of small, yet significant changes in body composition of resistancetrained male athletes. Given that change in physique is likely to be small in this population, the use of DXA, BOD POD, or SA is recommended.
\end{abstract}

Keywords: anthropometry, bioelectrical impedance spectroscopy, BOD POD, dual-energy X-ray absorptiometry, fat-free mass, fat mass, least significant change

The association between athletic physique traits and competitive sporting success is well established (Meyer et al., 2013; Olds, 2001). In sports requiring high-force production, athletes with high levels of muscularity can gain a competitive advantage (Bilsborough et al., 2016; Gabbett, 2009; Olds, 2001), yet these athletes tend to see only small adaptations or improvements in physique over time (Binkley et al., 2015; Harley et al., 2011; Lees et al., 2017; Smart et al., 2013). Given this evidence, assessment methods with high precision are required to measure body composition on a regular basis in these athletes. By accurately quantifying changes in physique, more refined training and dietary interventions may be implemented, which can positively influence performance outcomes (Slater et al., 2005).

A variety of body composition assessment methods are available to quantify fat-free mass (FFM) and fat mass (FM) (Ackland et al., 2012; Kerr et al., 2017). Depending on time and resources, the four most popular methods used on athletic populations are air displacement plethysmography (BOD POD), dual-energy X-ray absorptiometry (DXA), bioelectrical impedance spectroscopy (BIS), and surface anthropometry (SA) (Meyer et al., 2013). Despite differences in technology, resources, and technical expertise required, they are all susceptible to technical error and

Farley and Slater are with the School of Health and Sport Sciences, University of the Sunshine Coast, Sippy Downs, Queensland, Australia. Hind is with the Department of Sport and Exercise Sciences, Durham University, Durham, United Kingdom. Farley (afarley@usc.edu.au) is corresponding author. biological variation (Ackland et al., 2012; Meyer et al., 2013), which significantly affects precision (Kerr et al., 2017; Kerr et al., 2018). Technical error is influenced by quality control procedures, such as subject clothing (Fields et al., 2000; Vescovi et al., 2002), and positioning during assessment (Kerr et al., 2016; Lambrinoudaki et al., 1998; Tegenkamp et al., 2011), level of technical expertise (Hume \& Marfell-Jones, 2008; Ruiz et al., 1971), and equipment calibration (Marfell-Jones et al., 2012). Biological variation may result from food and fluid ingestion or exercise prior to assessment and appears to influence most body composition methods, albeit to different degrees (Bone et al., 2017; Kerr et al., 2017). Other biological variables known to have an impact on estimates of body composition include body temperature and skin moisture (Fields et al., 2004), gastrointestinal contents (Bone et al., 2017), and muscle solutes (Rouillier et al., 2015).

While quantification of precision error $(\mathrm{PE})$ is frequently done by calculating differences in same-day repeat assessments of body composition (Hangartner et al., 2013; Hind et al., 2018) using least significant change (LSC) values, this fails to account for biological variability in the absence of controls, and can be evident during longitudinal monitoring (Meyer et al., 2013). Given this evidence, we have advocated for identifying the LSC for same-day (technical error) and consecutive-day (biological variation) precision in estimates of FM and FFM, finding a more accurate interpretation of true and meaningful change (Zemski et al., 2019). Currently, the precision of BOD POD, BIS, and SA, using LSC values for sameday and consecutive-day analysis, has not been explored. Therefore, the aims of this study were to (a) establish the same-day 
technical error of the four methods and (b) determine the consecutive-day PE of the methods using LSC values to determine the threshold of meaningful change in resistance-trained male athletes.

\section{Methods}

\section{Subjects}

Thirty-two White volunteers participated in this study and met the inclusion criteria, which included being male, having at least $\geq 2$ years resistance training experience, and having a body mass index of $\geq 25$. Subjects were excluded from the study if they were $>190 \mathrm{~cm}$ tall due to the limitation of the active scanning area of the DXA bed. The characteristics of all individuals are presented in Table 1. All subjects were informed of the nature and possible risks of the investigation before giving their written informed consent. This study was conducted according to the guidelines laid down in the Declaration of Helsinki, and all procedures involving subjects were approved by the human research ethics committee of the University of the Sunshine Coast (ethics approval no. S/12/450).

\section{Experimental Design}

Each subject underwent three testing sessions during a 24-hr window over a 2-day period (Figure 1), with each measurement taken by the same technician. The sessions commenced with body mass and stretch stature measured in minimal clothing, a total body DXA scan immediately followed by a BIS estimation of total body water (TBW), a BOD POD test, and an assessment of subcutaneous FM via the skinfold technique, in that sequence. Each subject undertook Tests 1 (D1T1) and 2 (D1T2) on Day 1 under standardized conditions (early morning, overnight fasted, well rested, and bladder voided). D1T2 was undertaken immediately after D1T1, and Test 3 (D2) was undertaken the following morning, $24 \mathrm{hr}$ after D1T1. Comparison of these testing sessions allowed for the calculation of typical error of measurement, random consecutive-day biological variability, and the difference in estimates of body composition data.

\section{Subject Presentation}

Guidance was provided on both days to encourage adherence to the standardized presentation for all three of the tests (D1T1, D1T2, and D2), as per current best practice guidance (Fields et al., 2004; Hind et al., 2018; Hume et al., 2018; Kyle et al., 2004). Subjects were required to present overnight fasted, bladder voided, and well rested (no prior physical activity) on the mornings before D1T1 and D2. They were asked to wear minimal fitted clothing, with metal objects and jewelry removed, plus the clothing was checked for metal zips or studs. Hydration status was assessed by a midstream sample of urine provided by the subjects early on both mornings before testing (D1T1 and D2). The specific gravity of the urine sample was measured using a digital refractometer (UG-Alpha; Atago Corporation, Tokyo, Japan). All subjects voided their bladder before tests.

\section{Dual-Energy X-Ray Absorptiometry}

All DXA scans were undertaken in the total body mode on a pencil beam DXA scanner (Lunar DPX; GE Healthcare, Madison, WI), with analysis performed using GE enCORE software (version 13.60; GE Healthcare) with the combined Geelong/Lunar reference database. Coefficient of variation (CV) measurements for the laboratory being $0.1 \%, 2.2 \%, 0.6 \%$, and $1.0 \%$ for BM, FM, lean mass, and bone mineral content, respectively. The DXA was calibrated with phantoms, as per the manufacturer's

Table 1 Descriptive Statistics for Body Composition Variables

\begin{tabular}{|c|c|c|c|c|}
\hline \multirow[b]{2}{*}{ Variable } & \multicolumn{2}{|c|}{ Day 1} & \multicolumn{2}{|c|}{ Day 2} \\
\hline & $n=32$ & Range & $n=32$ & Range \\
\hline Age (years) & $31 \pm 7$ & $18-47$ & & \\
\hline Stature $(\mathrm{cm})$ & $182.5 \pm 7$ & $168.7-190.0$ & & \\
\hline Mass (kg) & $91.5 \pm 10.1$ & $75.1-114.5$ & $91.5 \pm 10.1$ & $75.1-113.9$ \\
\hline BMI $\left(\mathrm{kg} / \mathrm{m}^{2}\right)$ & $27 \pm 3$ & $25-33$ & & \\
\hline USG & $1.019 \pm 0.007$ & $1.002-1.028$ & $1.019 \pm 0.007$ & $1.002-1.029$ \\
\hline BMC (kg) & $3.84 \pm 0.42$ & $2.89-4.67$ & $3.84 \pm 0.42$ & $2.91-4.67$ \\
\hline TBW (kg) & $57.3 \pm 5.8$ & $47.1-71.1$ & $57.1 \pm 6.1$ & $47.0-71.4$ \\
\hline Skinfolds sum of $8(\mathrm{~mm})$ & $82 \pm 30$ & $36-172$ & $81 \pm 30$ & $36-171$ \\
\hline \multicolumn{5}{|l|}{ FM (kg) } \\
\hline BOD POD & $16.8 \pm 6.4$ & $6.4-32.0$ & $16.6 \pm 6.3$ & $6.5-31.3$ \\
\hline BIS & $13.3 \pm 7.2$ & $1.1-31.0$ & $13.4 \pm 6.6$ & $2.0-30.9$ \\
\hline DXA & $17.1 \pm 6.9$ & $5.0-33.4$ & $16.8 \pm 6.6$ & $5.8-32.3$ \\
\hline SA & $11.0 \pm 4.2$ & $5.2-22.9$ & $10.9 \pm 4.1$ & $5.1-22.5$ \\
\hline \multicolumn{5}{|l|}{ FFM (kg) } \\
\hline BOD POD & $74.8 \pm 7.5$ & $60.7-90.0$ & $75.0 \pm 7.5$ & $61.7-91.0$ \\
\hline BIS & $78.2 \pm 7.9$ & $64.4-97.1$ & $78.1 \pm 8.3$ & $64.2-97.6$ \\
\hline DXA & $74.7 \pm 7.6$ & $60.0-91.3$ & $74.9 \pm 7.8$ & $59.8-93.0$ \\
\hline $\mathrm{SA}$ & $80.6 \pm 8.2$ & $68.4-102.5$ & $80.7 \pm 8.2$ & 68.3-102.1 \\
\hline
\end{tabular}




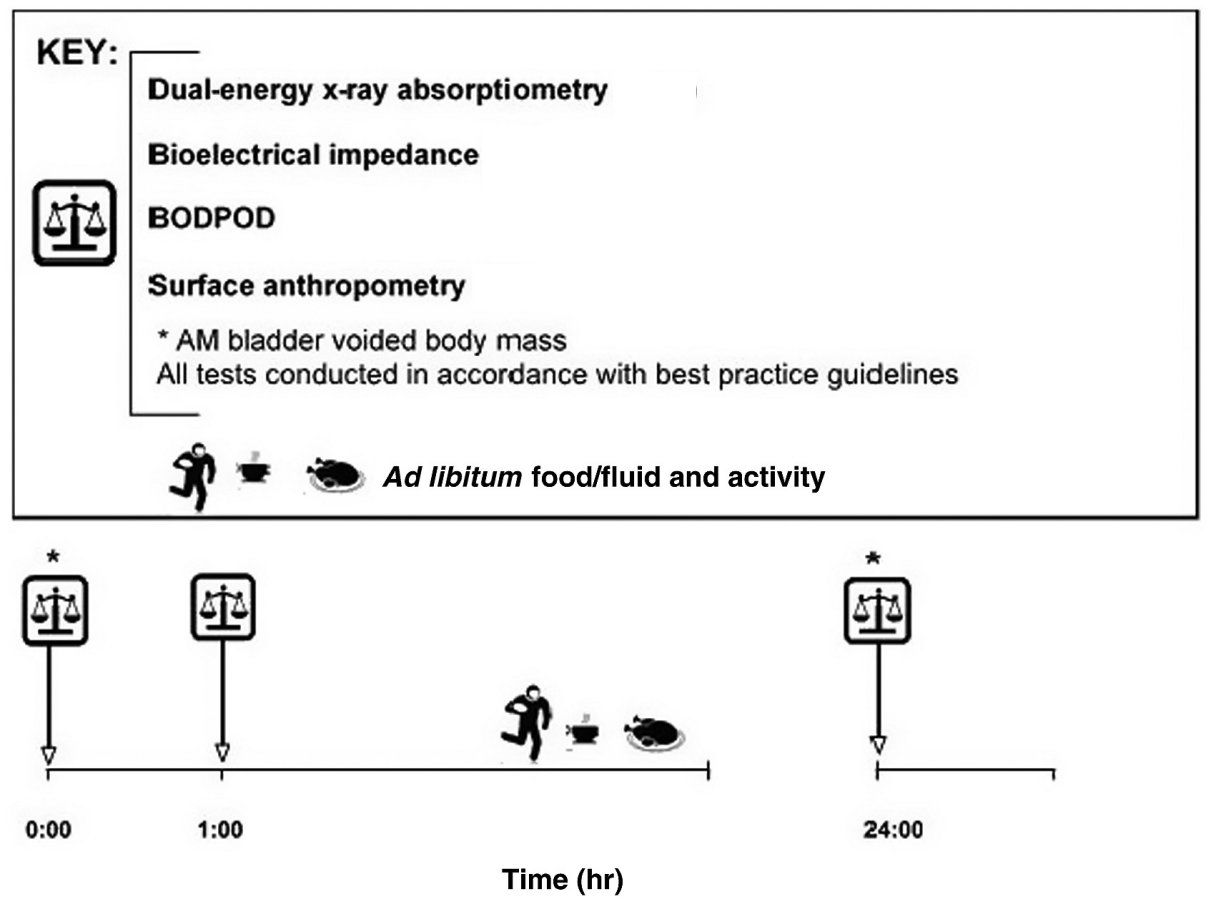

Figure 1 - Study design of three testing sessions conducted over $24 \mathrm{hr}$.

guidelines, each day before the measurements were taken. All scans were conducted by the same Queensland Radiation Health licensed technician, using the standard thickness mode as determined by the auto scan feature in the software, and all safety protocols were adhered to as per the University of the Sunshine Coast Radiation Safety Protection Plan. The scans were performed according to a protocol developed that emphasized a consistent positioning of subjects on the DXA scanning bed (Nana et al., 2012a), as previously described (Nana et al., 2012b). In addition, two Velcro straps were used to minimize any subject movement during the scan as well as to provide a consistent body position for subsequent scans. One strap was secured around the ankles above the foot positioning pad, and the other strap was secured around the trunk at the level of the mid forearms (Kerr et al., 2016). All scans were analyzed automatically by the DXA software, but all regions of interest were reconfirmed before being included in the subsequent statistical analysis.

\section{Bioelectrical Impedance Spectroscopy}

Immediately after each DXA scan, while the subjects were still positioned on the DXA scanning bed, body composition, derived from TBW obtained values, was measured using the SFB7 BIS device (ImpediMed Ltd., Brisbane, Queensland, Australia). Subject positioning was standardized (Kyle et al., 2004) to ensure supine positioning on the nonconductive foam mattress without contact to the metal side supports of the DXA scanner for a minimum of 15 min before BIS measurements (Ward et al., 2015). The BIS was calibrated as per the manufacturer's instructions, with each participant's stature, body mass, age, and sex programmed into the unit. Sites of attachment for the electrodes (ImpediMed) were first shaved and cleaned with alcohol wipes before the dual-tab electrodes were attached as follows: one electrode was attached centrally on the top side of the wrist in alignment with the ulnar head and $5 \mathrm{~cm}$ lower on the dorsal surface of the hand. The second electrode was attached centrally on the dorsal surface of the ankle between the lateral and medial malleoli and $5 \mathrm{~cm}$ lower on the dorsal surface of the foot, which is in accordance with previous guidelines (Kerr et al., 2015). The SFB7 measures impedance using 256 frequencies between 4 and $1024 \mathrm{kHz}$ to estimate TBW based on a Cole-Cole plot (Cornish et al., 1996). Three measurements were taken consecutively, and the median of these used in subsequent analysis. The TBW value, as per the Pace et al. model (Pace \& Rathbun, 1945), was used to estimate body composition of FFM and FM by simple subtraction from body mass.

\section{Air Displacement Plethysmography}

Immediately after BIS measurement, assessment of body density was undertaken using the BOD POD (life measurement instruments) following the recommended procedures of the manufacturer (Dempster \& Aitkens, 1995), utilizing a validated, predicted thoracic lung volume estimation (McCrory et al., 1998). Subjects wore Lycra clothing and a silicone swim cap, with all metal objects removed before measurement. Body density was calculated by the BOD POD's software system (version 5.3.2; COSMED, Concord, CA) as follows:

$$
D(\text { density }) \operatorname{Mass}(\text { scale })=\text { Volume }(\text { BOD POD }) \text {. }
$$

An estimate of FM and FFM was obtained to calculate \%BF, as defined by the Siri equation (Siri, 1961), as follows:

$$
\% B F=(497.1 / \text { body density })-451.9 \text {. }
$$

\section{Surface Anthropometry}

Immediately after completion of the BOD POD assessment, duplicate skinfold measurements were taken, according the International Society of the Advancement of Kinanthropometry technique, by 
Table 2 Mean Difference $( \pm S D)$ Between Same-Day Tests (Technical Error) and Consecutive-Day Tests (Technical Error and Biological Variation)

\begin{tabular}{lcc}
\hline & $\begin{array}{c}\text { Same day } \\
n=32\end{array}$ & \begin{tabular}{c} 
Consecutive day \\
\cline { 3 - 3 } D1T1/D1T2
\end{tabular} \\
\hline FM (g) & D1T1/D2 \\
BIS & $676 \pm 698$ & $1,132 \pm 988$ \\
BOD POD & $483 \pm 360$ & $642 \pm 492$ \\
DXA & $376 \pm 332$ & $520 \pm 426$ \\
SA & $174 \pm 171$ & $147 \pm 110$ \\
FFM (g) & & \\
BIS & $663 \pm 679$ & $1,234 \pm 1097$ \\
BOD POD & $478 \pm 368$ & $620 \pm 487$ \\
DXA & $493 \pm 355$ & $587 \pm 572$ \\
SA & $168 \pm 167$ & $381 \pm 295$ \\
\hline
\end{tabular}

Note. Values are presented as mean $\pm S D$. D1T1 = Day 1 Test 1 ; D1T2 $=$ Day 1 Test 2; D2 = Day 2 Test 1; FM = fat mass; FFM = fat-free mass; BOD POD = air displacement plethysmography; $\mathrm{BIS}=$ bioelectrical impedance spectroscopy; DXA = dual-energy X-ray absorptiometry; SA = surface anthropometry.
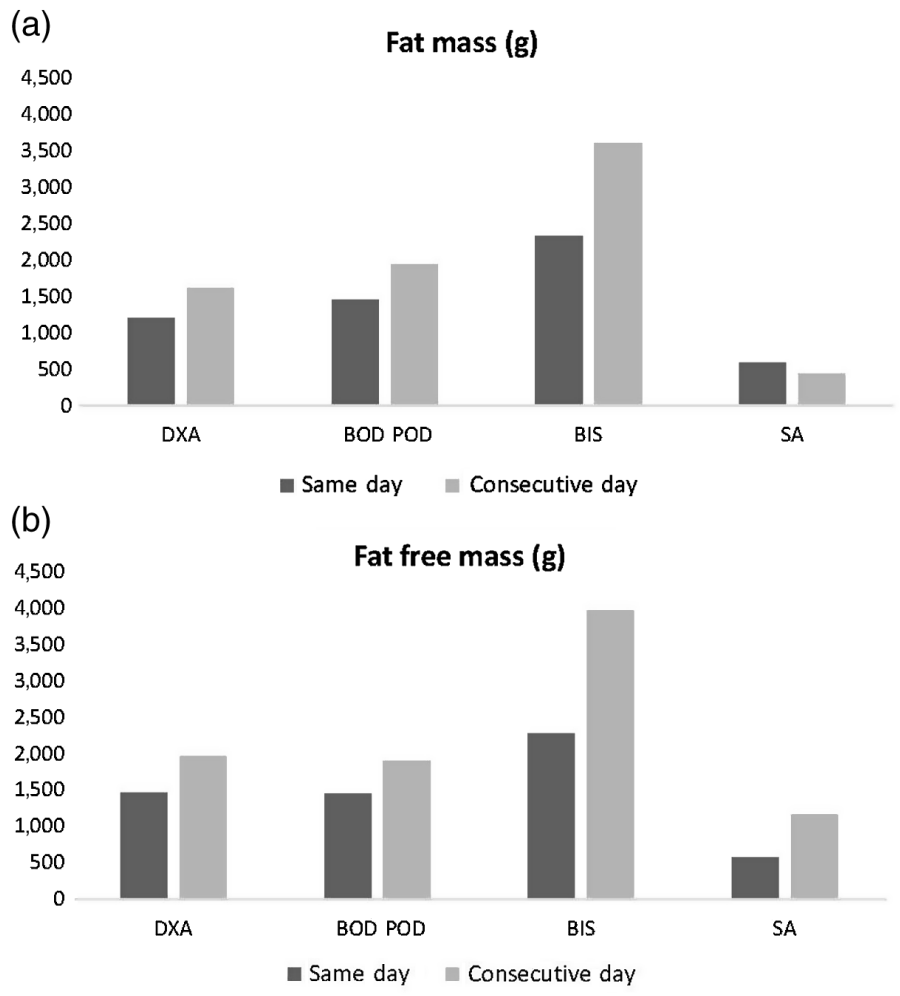

Figure 2 - LSC for DXA, BOD POD, BIS, and SA for same-day and consecutive-day measures. BOD $\mathrm{POD}=$ air displacement plethysmography; $\mathrm{BIS}=$ bioelectrical impedance spectroscopy; DXA = dual-energy X-ray absorptiometry; $\mathrm{SA}=$ surface anthropometry; $\mathrm{LSC}=$ least significant change.

the same technician certified by the International Society of the Advancement of Kinanthropometry, as previously described (Norton et al., 1996).

The sum of eight skinfolds was determined following measurements of the triceps, biceps, subscapular, iliac crest, supraspinale, abdominal, quadriceps, and calf skinfolds using a
Table 3 Precision Error for Each Method, Represented as the \%CV, With the RMS-SD, LSC, and \%LSC

\begin{tabular}{lcccc}
\hline $\begin{array}{l}\text { Method } \\
\begin{array}{l}\text { D1T1/D1T2 technical error } \\
\text { FM (g) }\end{array}\end{array} \begin{array}{c}\text { RMS- } \\
\text { SD }\end{array}$ & LSC-95\% Cl & $\begin{array}{c}\text { \% } \\
\text { CV }\end{array}$ & \%LSC-95\% Cl \\
BIS & 842 & 2,331 & 5.2 & 14.4 \\
BOD POD & 523 & 1,448 & 2.5 & 6.9 \\
DXA & 435 & 1,204 & 1.5 & 4.2 \\
SA & 212 & 586 & 1.0 & 2.9 \\
FFM (g) & & & & \\
BIS & 822 & 2,276 & 0.6 & 1.6 \\
BOD POD & 524 & 1,450 & 0.5 & 1.3 \\
DXA & 528 & 1,461 & 0.5 & 1.3 \\
SA & 205 & 568 & 0.2 & 0.4
\end{tabular}

D1T1/D2 technical error and biological variation

FM (g)

$\begin{array}{lcccc}\text { BIS } & 1,302 & 3,607 & 9.4 & 25.9 \\ \text { BOD POD } & 702 & 1,943 & 2.8 & 7.8 \\ \text { DXA } & 583 & 1,615 & 2.4 & 6.6 \\ \text { SA } & 160 & 442 & 1.0 & 2.7 \\ \text { FFM (g) } & & & & \\ \text { BIS } & 1,432 & 3,966 & 1.1 & 3.1 \\ \text { BOD POD } & 684 & 1,894 & 0.6 & 1.7 \\ \text { DXA } & 710 & 1,967 & 0.5 & 1.5 \\ \text { SA } & 418 & 1,159 & 0.3 & 0.9\end{array}$

Note. D1T1 = Day 1 Test 1 ; D1T2= Day 1 Test 2; D2= Day 2 Test 1; RMS$S D=$ root mean square $S D ; \mathrm{LSC}=$ least significant change; $\mathrm{CV}=$ coefficient of variation; $\mathrm{CI}=$ confidence interval; $\mathrm{BIS}=$ bioelectrical impedance spectroscopy; BOD $\mathrm{POD}=$ air displacement plethysmography; $\mathrm{DXA}=$ dual-energy $\mathrm{X}$-ray absorptiometry; $\mathrm{SA}=$ surface anthropometry; $\mathrm{FM}=$ fat mass; $\mathrm{FFM}=$ fat-free mass.

calibrated skinfold caliper (Harpenden; Baty International, Burgess Hill, United Kingdom). Due to the similar procedure, equipment, and population used, the 4C validated Evans equation of three skinfolds (triceps, abdominal, and thigh) was utilized to calculate $\% \mathrm{BF}$ as (Evans et al., 2005):

$$
\begin{aligned}
\% \mathrm{BF}= & 8.997+0.24658 \times(3 \mathrm{SKF})-6.343 \times(\text { gender })-1.998 \\
& \times(\text { race }),
\end{aligned}
$$

Gender coded as $0=$ female, $1=$ male, and ethnicity coded as $0=$ White, $1=$ Black.

Stretch stature was measured with a stadiometer (Harpenden; Holtain Ltd., Crymych, United Kingdom) to the nearest $0.1 \mathrm{~cm}$. Body mass was measured on a calibrated scale to the nearest $0.01 \mathrm{~kg}$ (Seca GmbH, Hamburg, Germany).

\section{Statistical Analysis}

Data analysis was performed using Microsoft Excel (Microsoft, Redmond, WA). Descriptive data are reported as the mean $\pm S D$. The precision is reported as the root mean square $S D$ and $\% \mathrm{CV}$. The resulting LSC with 95\% confidence intervals was calculated following the International Society for Clinical Densitometry (ISCD) protocol (Hangartner et al., 2013). The \%CV was derived from the equation $\% C V=(S D /$ mean $) \times 100$. Coefficients of determination $\left(R^{2}\right)$ were calculated for measurements to establish the relationship 

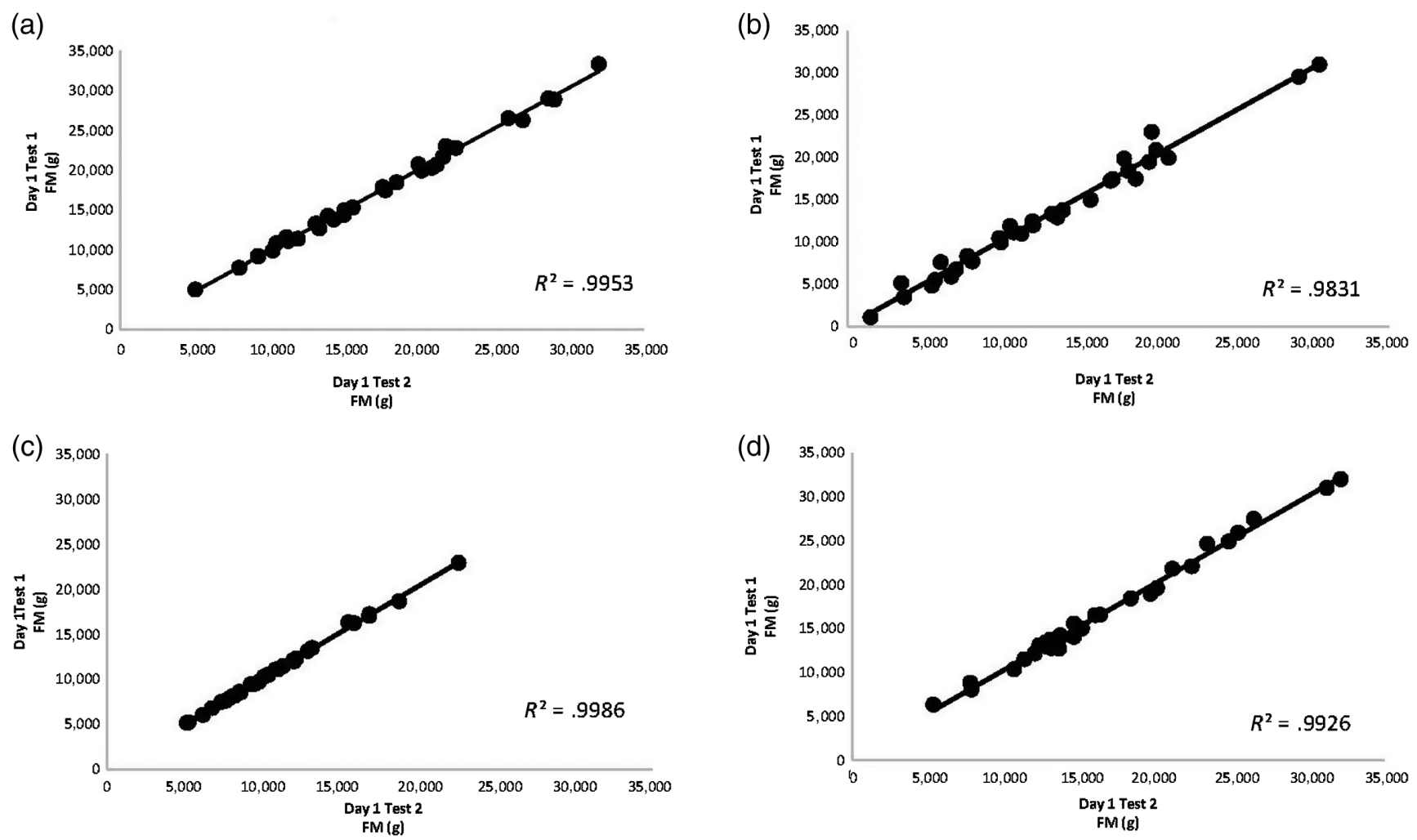

Figure 3 - Regression analysis between measures of FM for same-day precision. FM indicates fat mass.

(a)

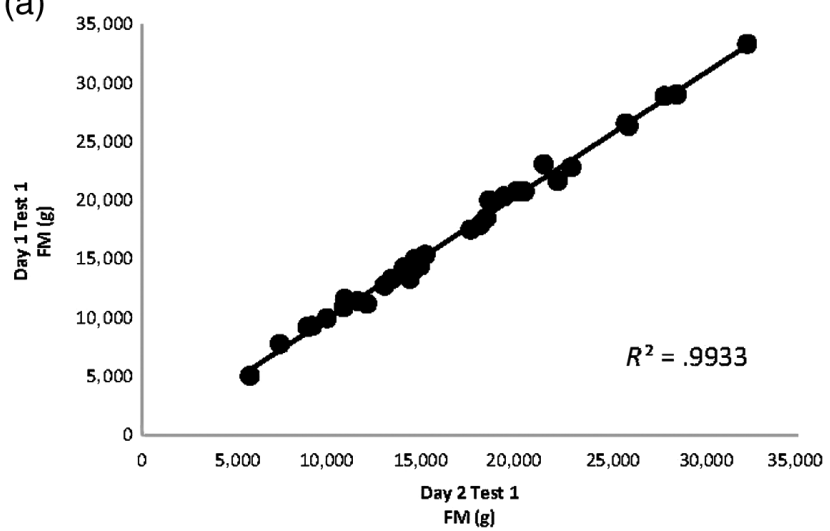

(c)

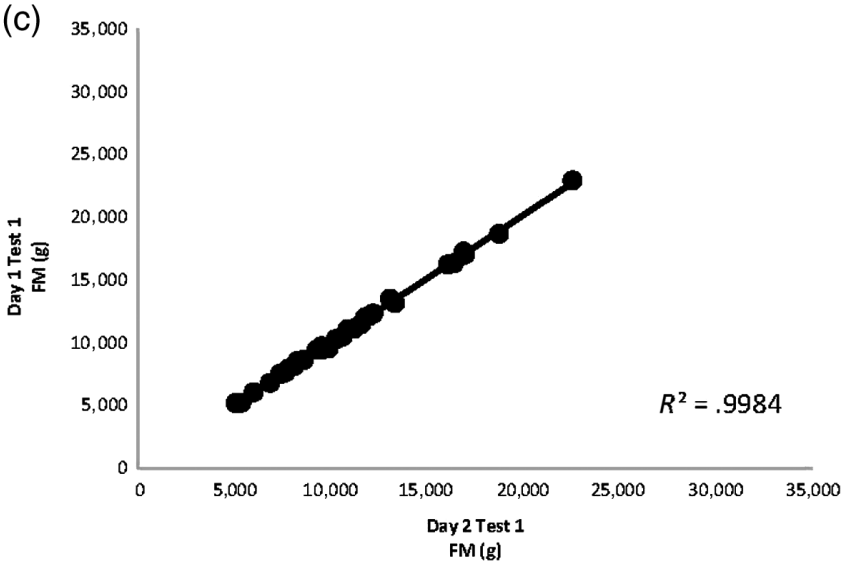

(b)

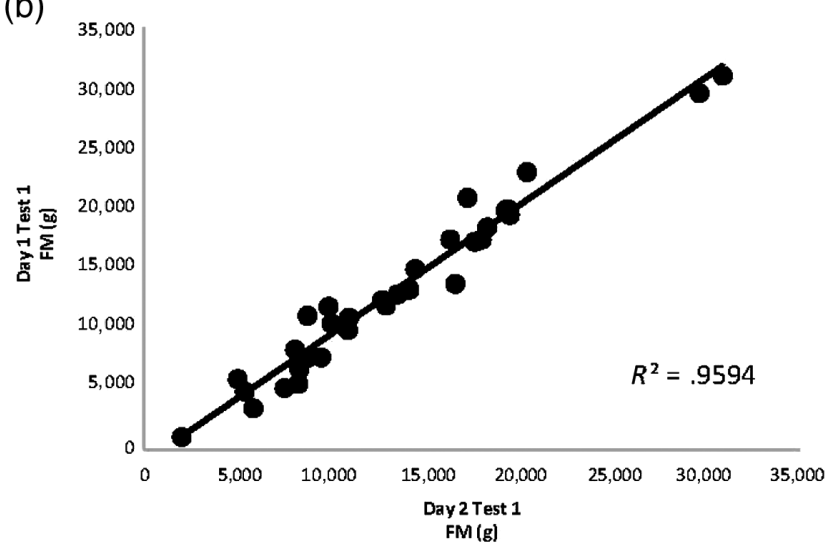

(d)

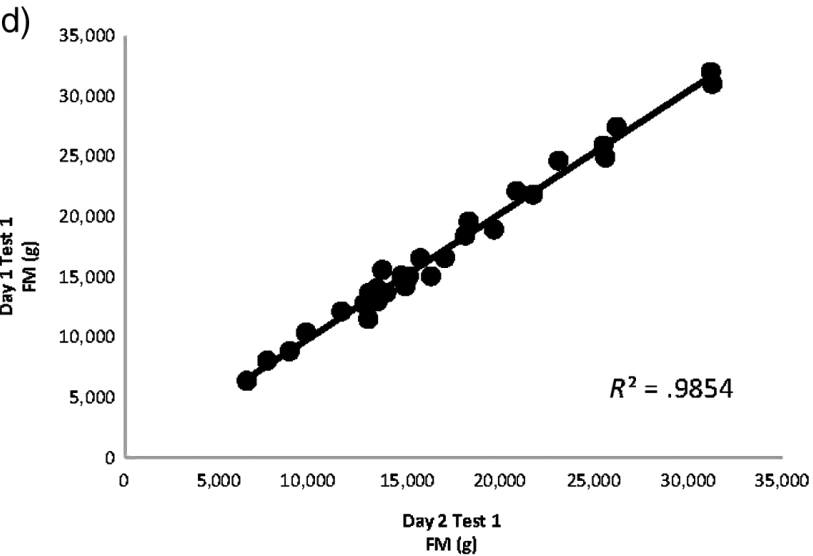

Figure 4 - Regression analysis between measures of FM for consecutive-day precision. FM indicates fat mass. 
between same-day and consecutive-day measures. A one-way analysis of variance was used to determine if there was an effect of time on FM and FFM for each method. Statistical significance was set at .05. Bland-Altman plots were created to compare individual same-day and consecutive-day precision for all techniques.

\section{Results}

Descriptive statistics for the participants in this study are given in Table 1 . The analysis of variance showed there was no effect of time on FM, $F(2,93)=0.01-0.03, p>.97$, and FFM, $F(2,93)=$ $0.01-0.02, p>.99$, for each method. The mean differences between same-day (technical error) and consecutive-day (technical error and biological variation) testing for FM and FFM in all methods are shown in Table 2. Differences between same-day and consecutiveday testing, demonstrating the LSC values for all methods, are given in Figure 2.

Table 3 shows the PE for each method of testing, represented as the $\% \mathrm{CV}$, with the root mean square SD, LSC, and \%LSC. Strong agreement was found for all methods for same-day and for consecutive-day FM regression analysis (SA $R^{2}=1.00-1.00$, BOD POD $R^{2}=.99-.99$, DXA $R^{2}=1.00-.99$, BIS $R^{2}=.98-.96$ ), as shown in Figures 3 and 4. Regression analysis undertaken for same-day and consecutive-day FFM for all methods revealed strong relationships (SA $R^{2}=1.00-1.00$, BOD POD $R^{2}=.99-.99$, DXA $R^{2}=.99-.99$, BIS $\left.R^{2}=.99-.96\right)$, as shown in Figures 5 and 6 .

Bland-Altman analysis revealed that SA had the smallest level of bias between same-day and consecutive-day precision for FM $(83 \mathrm{~g})$ and FFM (178 g), with very low limits of agreement $(\mathrm{FM}=$
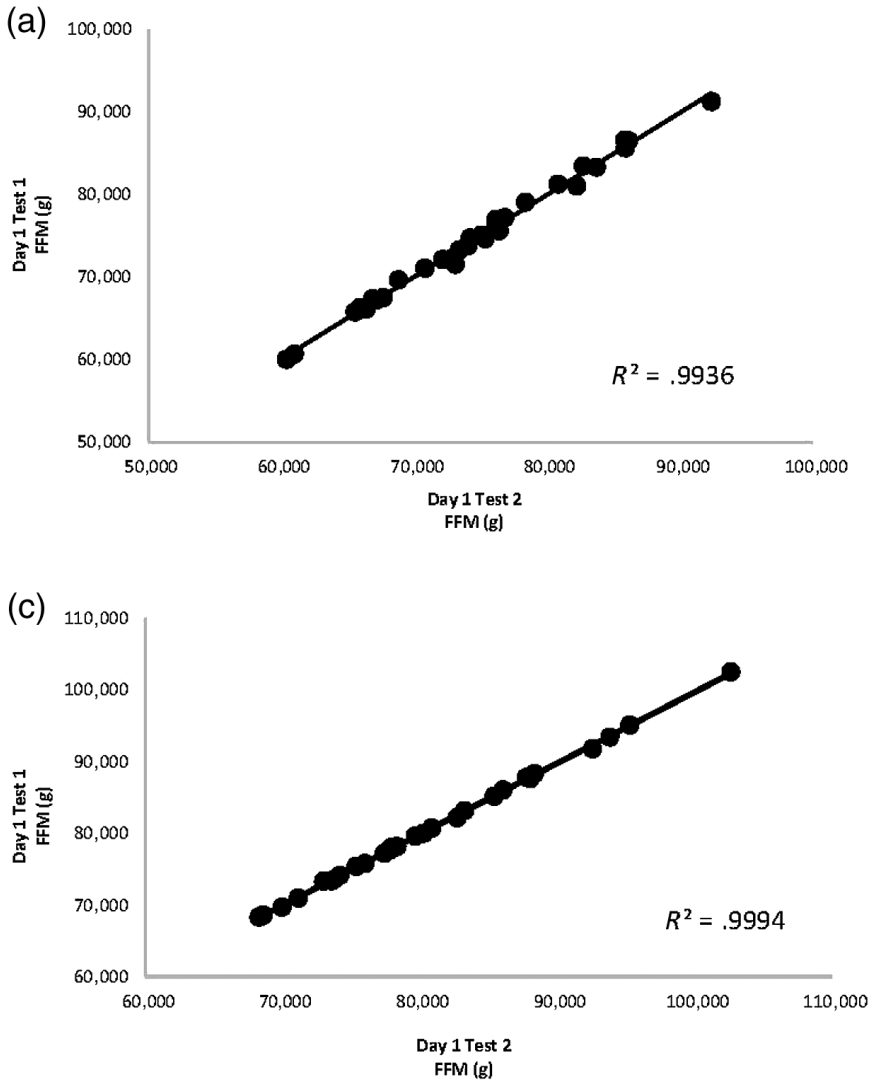

-7 to $173 \mathrm{~g}$; FFM $=-185$ to $172 \mathrm{~g}$ ). DXA and BOD POD had low levels of bias between same-day and consecutive-day precision for FM $(\mathrm{DXA}=226 \mathrm{~g}$; BOD POD = $318 \mathrm{~g})$ and FFM $(\mathrm{DXA}=309 \mathrm{~g}$; $\mathrm{BOD} P O D=321 \mathrm{~g})$, with low limits of agreement for DXA $(\mathrm{FM}=$ -365 to $87 \mathrm{~g} ; \mathrm{FFM}=-59$ to $558 \mathrm{~g}$ ) and BOD POD (FM $=-275$ to $361 \mathrm{~g} ; \mathrm{FFM}=-251$ to $390 \mathrm{~g}$ ). The largest level of bias between same-day and consecutive-day precision came from BIS for FM $(524 \mathrm{~g})$ and FFM (580 g), with wider limits of agreement (FM = -108 to $939 \mathrm{~g}$; FFM $=-930$ to $230 \mathrm{~g}$ ), as shown in Figures 7 and 8.

\section{Discussion}

To our knowledge, this is the first study exploring both technical error and the short-term biological variation within a 24-hr period, using four independent methods of body composition assessment. The body composition PE was greater when quantified from consecutive-day compared with same-day results on a resistance-trained athletic male cohort. This was evident across all body composition assessment techniques. Consecutive-day PE was 25\% higher for DXA and BOD POD (FM and FFM) estimations and nearly 50\% higher for BIS (FM and FFM) and SA (FFM) than same-day PE. It must be noted that same-day and consecutiveday PE in SA (FFM) were lower than all other methods. In contrast, the SA FM PE for same-day and consecutive-day analysis was lower for consecutive day, but only by $8 \%$, and was not significantly different $(p<.5)$. This shows that biological variation affects measurement precision even within very short time frames $(24 \mathrm{hr})$, at least when using BIS, DXA, and BOD POD methodology. Therefore, the use of consecutive-day $\mathrm{PE}$ is advocated as

(b)
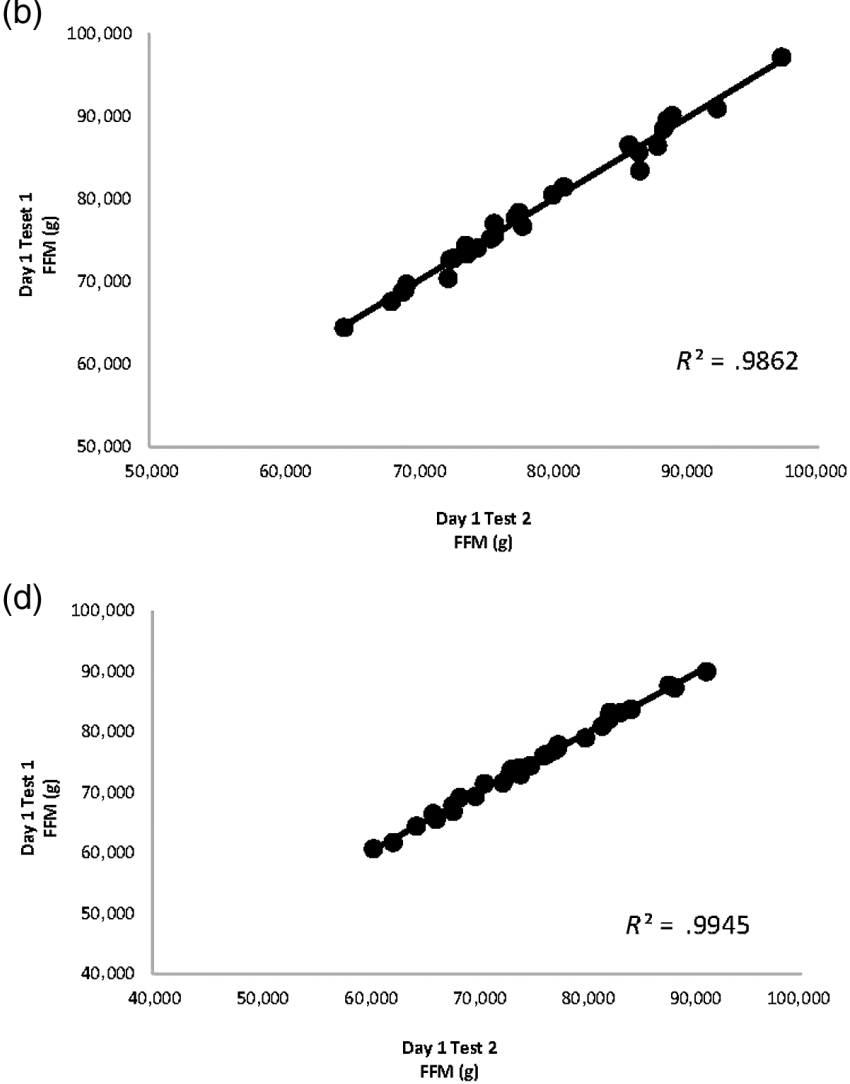

Figure 5 - Regression analysis between measures of FFM for same-day precision. FFM indicates fat-free mass. 
(a)

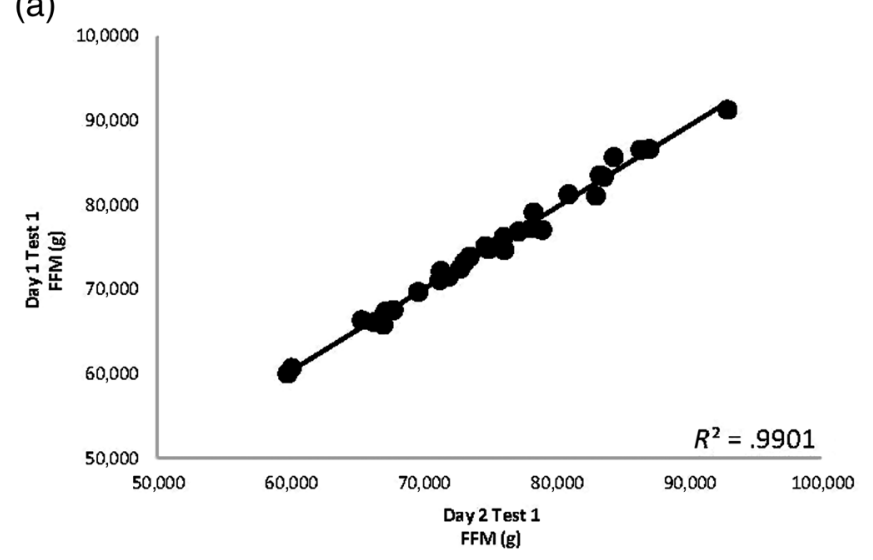

(c)

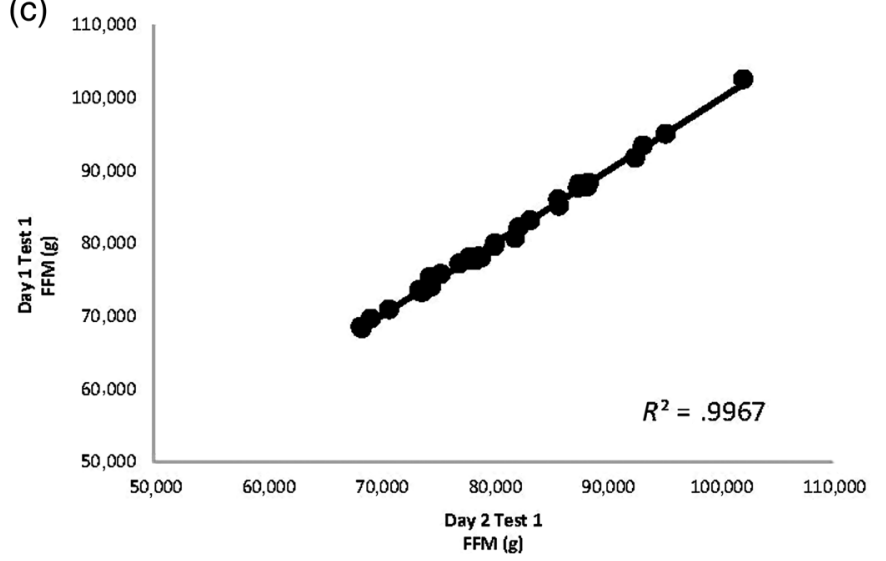

(b)

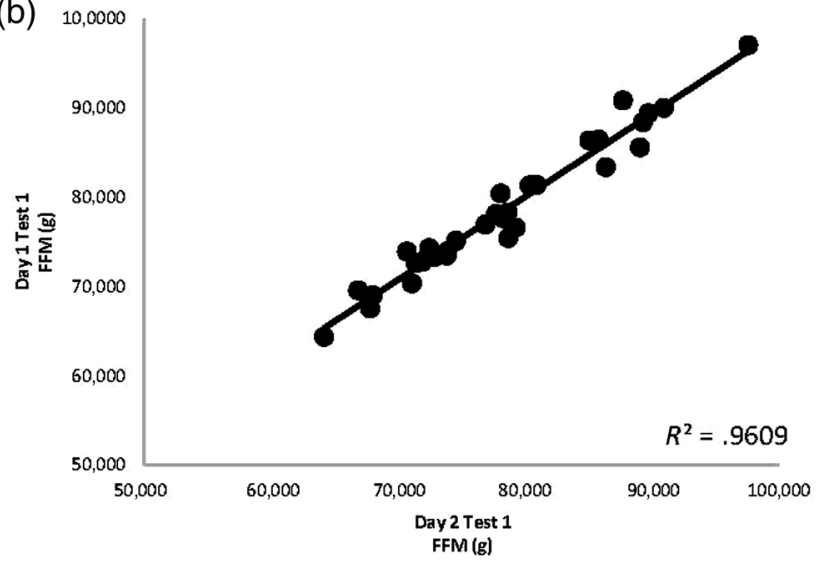

(d)

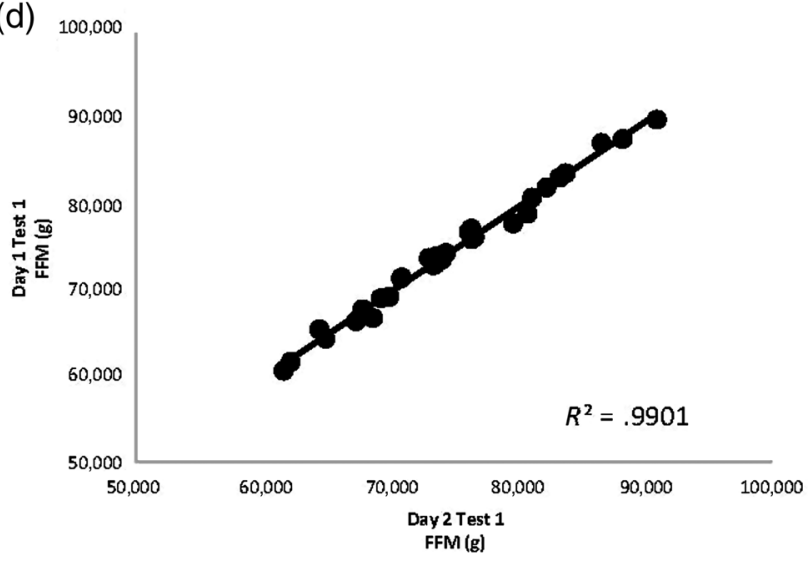

Figure 6 - Regression analysis between measures of FFM for consecutive-day precision. FFM indicates fat-free mass.

longitudinal monitoring of physique and will always include both technical error and biological variation.

Excellent SA same-day precision was found for estimations of FM (CV 1.0\%) and FFM (CV 0.2\%), as well as for consecutive-day testing with FM (CV 1.0\%) and FFM (CV 0.3\%), respectively. Raw measurements from SA (in millimeters) have been shown to be robust and unaffected by the biological variation caused by prior food and fluid ingestion or exercise (Kerr et al., 2017), yet this study included body mass to obtain estimates of FM and FFM using the Evans equation (Evans et al., 2005). It would be expected, then, that consecutive-day PE would be larger given that body mass is acutely influenced by hydration status, gastrointestinal tract contents, and muscle glycogen stores (Rouillier et al., 2015). Due to adopting previous recommendations of subject presentation, including overnight fasting, post bladder, and bowel evacuation with body measurements taken early in the morning in minimal clothing, the biological impact on precision was expected to be minimal (Kerr et al., 2017; Nana et al., 2015).

The DXA is prone to biological variance due to changes in hydration, significantly affecting FFM estimates (Kerr et al., 2017). This is particularly noticeable in large muscular males with high levels of FFM (Barlow et al., 2015; Bilsborough et al., 2014). Previous literature and manufacturing guidance suggest that a standardized testing protocol be adopted to minimize technical error and biological variation (Kerr et al., 2016; Nana et al., 2012a). This is in agreement with previously reported results, a CV of $0.5 \%$ and 1.5\%, respectively (De Lorenzo et al., 1997; Nana et al., 2012a), and more recently, results from Zemski et al. (2019), with a consecutive-day FM CV of $2.9 \%$ and lean mass CV of $1.1 \%$. Despite obtaining excellent precision from utilizing a standardized presentation protocol, those authors found biological variance (consecutive day) to be higher than technical error (same day), most probably due to short-term changes in hydration (Nana et al., 2012a), sleep hygiene (Vitale et al., 2019), and intramuscular solute levels (Bone et al., 2017). These findings would support the results from this study with an FM and FFM CV of $2.4 \%$ and $0.5 \%$, respectively. While current best practice guidance was followed, this may not account for variance in muscle solute content, which is known to influence reliability. The impact of standardized training and diet on consecutive-day precision warrants investigation.

Close comparisons between DXA and BOD POD were identified in this study, with strong agreement found in same-day and consecutive-day FM PE (BOD POD $R^{2}=.99-.99$, DXA $R^{2}=1.00$ 0.99 ) and FFM (BOD POD $R^{2}=.99-.99$, DXA $\left.R^{2}=.99-.99\right)$. In support, previous research using DXA and BODPOD technology shows consistent results with this study, with only small or trivial PE in FM and FFM estimates from consecutive-day testing conducted under standardized presentation conditions (Kerr et al., 2017). Despite BOD POD estimates of FM, and FFM being subject to biological variation if unrestricted subject presentation occurs (food and fluid intake plus physical activity), BOD POD precision in this study showed that very high resolution can be obtained if these variables are controlled (FM CV 2.8\%, FFM CV 0.6\%). A 

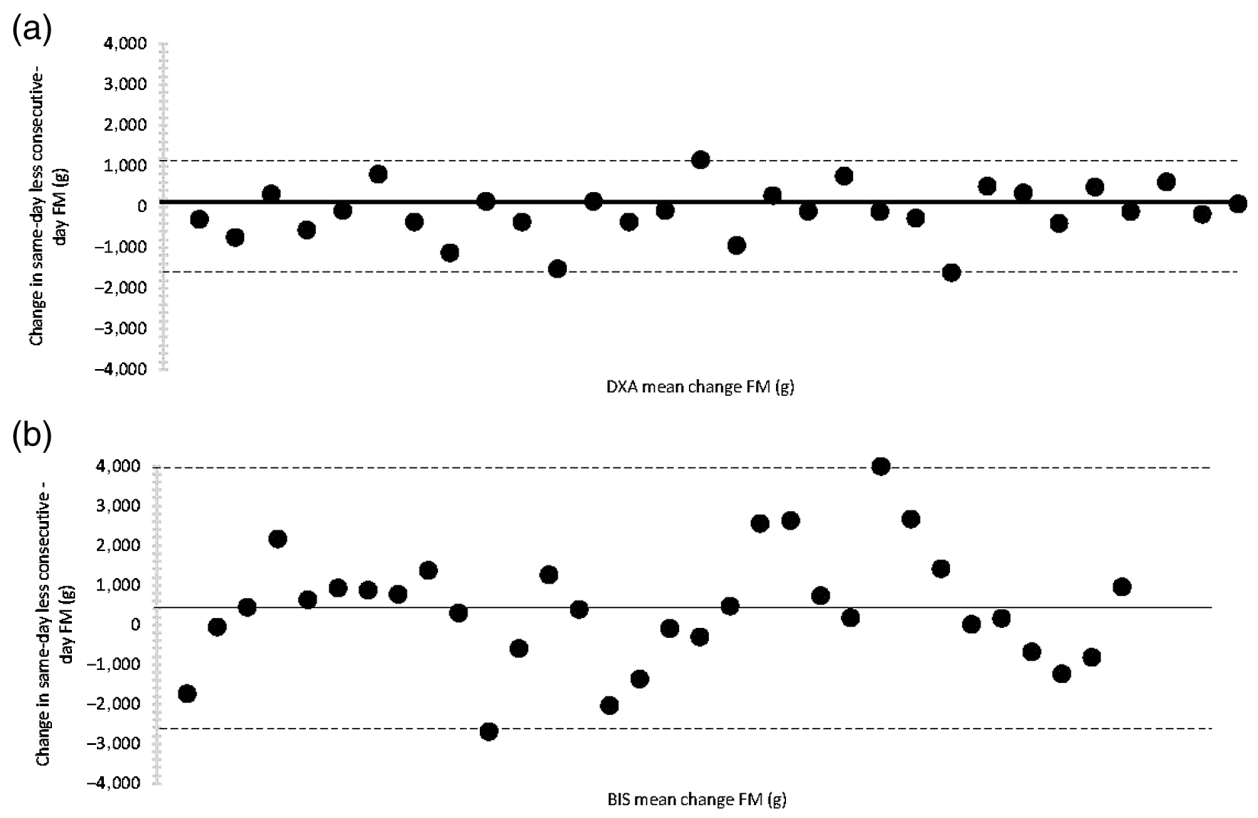

(c)

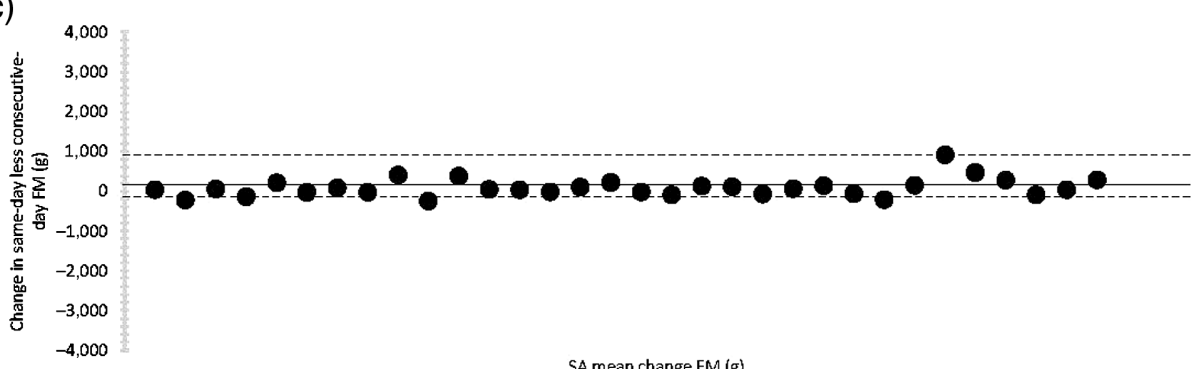

A mean change FM (E)

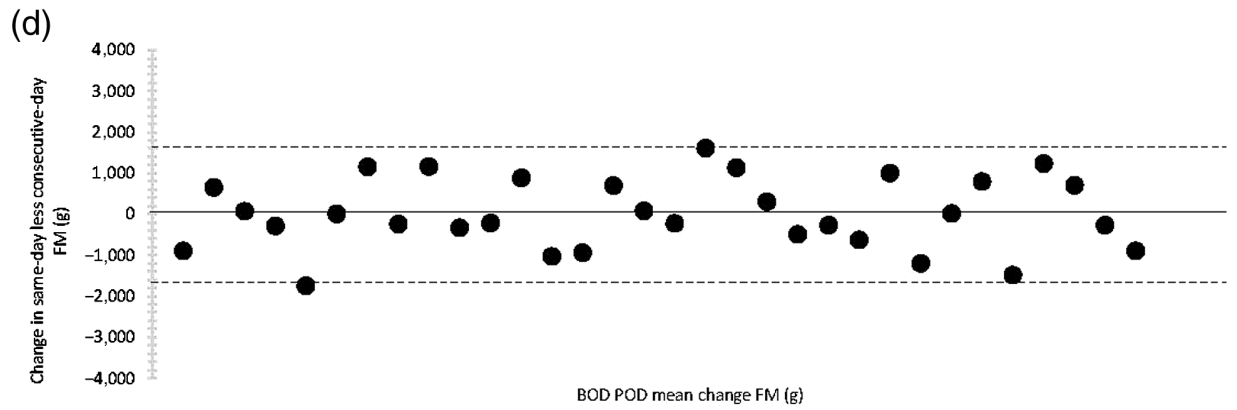

Figure 7 - Bland-Altman plots for differences in same-day versus consecutive-day measures for FM. FM indicates fat mass; BOD POD = air displacement plethysmography; BIS = bioelectrical impedance spectroscopy; DXA = dual-energy X-ray absorptiometry; SA = surface anthropometry.

limitation of this study is that the DXA scanner used to estimate body composition (GE Lunar DPX Pro) has been superseded by newer models with enhanced precision. PE from the DPX estimations has been found to be twice as high as the GE Lunar Prodigy in athletes (Bilsborough et al., 2014), whereas the iDXA model resolution has improved bone edge detection, thus allowing for superior algorithms for body composition estimation (Toombs et al., 2011).

Factors that impact TBW, such as prior food and fluid intake, physical activity, or medical conditions, make BIS vulnerable to imprecision (Kyle et al., 2004). Additionally, variance in fluid and electrolyte content will affect TBW (Saunders et al., 1998) and confound any change in physique traits inferred from BIS (O'brien et al., 2002). Given normal daily fluctuations in TBW, it is unsurprising that the change between same-day and consecutiveday precision using BIS derived estimates of FM and FFM showed nearly a $50 \%$ increase in PE for both FM (3,607 vs. 2,331 g) and FFM (3,966 g vs. 2,276 g) estimates. BIS also had the highest CV\% of all methods for both same-day FM and FFM (5.2\% and 0.6\%) and consecutive-day values $(9.4 \%$ and $1.1 \%)$, respectively. This suggests that despite implementing a rigorous athlete presentation protocol prior to testing, a lower tolerance level for precision still occurs. Given this evidence, the ability of BIS to accurately track small changes in the physique among athletic populations is questionable. 
(a)
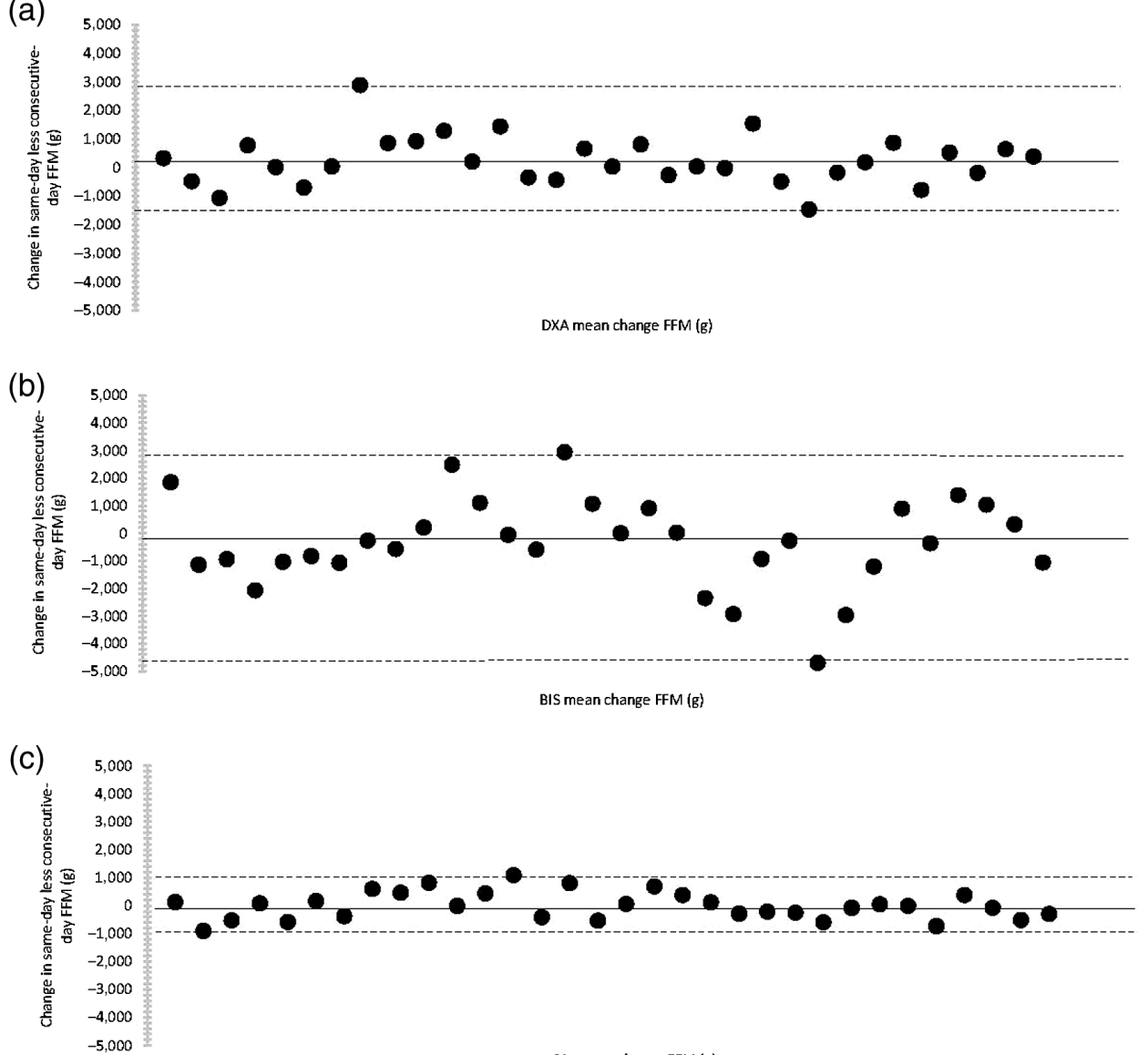

SA mean change FFM (g)

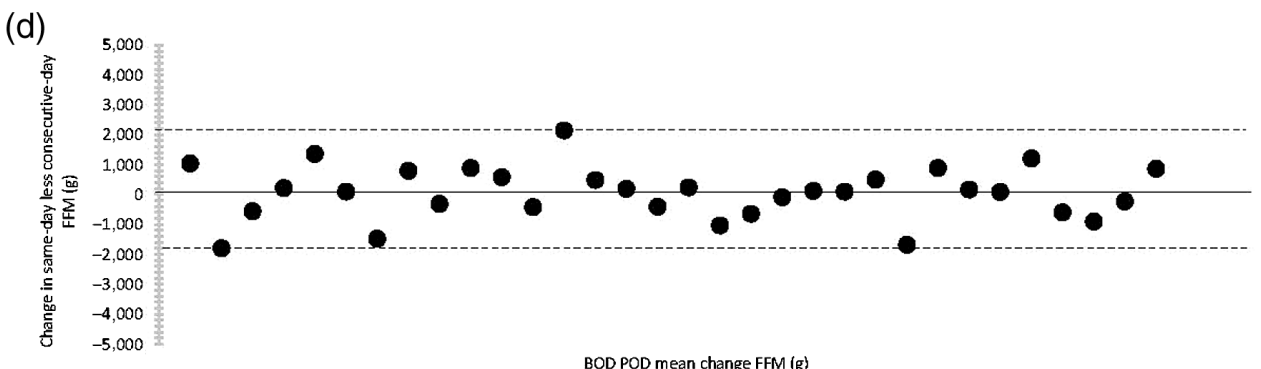

BOD POD mean change FFM (E)

Figure 8 - Bland-Altman plots for differences in same-day versus consecutive-day measures for FFM. FFM indicates fat-free mass; BOD POD = air displacement plethysmography; BIS = bioelectrical impedance spectroscopy; DXA = dual-energy X-ray absorptiometry; SA = surface anthropometry.

\section{Conclusion}

In conclusion, consecutive-day PE was larger than same-day for FM and FFM estimates obtained from DXA, BOD POD, SA, and BIS (except for SA FM, which was marginally lower) in a cohort of muscular resistance-trained male athletes. This is despite PE limits for FM and FFM estimates being within acceptable precision thresholds, at least for DXA. Clearly all methods are subject to some imprecision due to daily biological fluctuations, especially BIS, which calculates physique traits from a TBW estimation. Given that both technical error and biological variation contribute to precision, we recommend the use of LSC values calculated from consecutive-day analysis when interpreting longitudinal change for true changes in physique. Application of DXA, BOD POD, or SA should be advocated over BIS for athletic populations where only small changes are observed over time.

\section{Practical Implications}

Adopting LSC values from consecutive-day analysis likely provide a more appropriate benchmark to assess meaningful change in body composition of athletic populations longitudinally.

\section{Acknowledgments}

The authors' responsibilities were as follows-AF and G.J. Slater helped in the study concept and design; AF helped in the acquisition of data; $\mathrm{AF}$ and G.J. Slater assisted in analysis and interpretation of data; AF helped in drafting the manuscript; AF, G.J. Slater, and K. Hind assisted in the critical revision of the manuscript for important intellectual content; AF helped in statistical analysis; and G.J. Slater in study supervision. AF had full access to all the data in the study and takes responsibility for the integrity and the accuracy of the data analysis. The results of this study are presented clearly, 
honestly, and without fabrication, falsification, or inappropriate data manipulation. The authors have no financial or personal conflicts of interest to declare. There were no funding sources for the present study.

\section{References}

Ackland, T.R., Lohman, T.G., Sundgot-Borgen, J., Maughan, R.J., Meyer, N.L., Stewart, A.D., \& Müller, W. (2012). Current status of body composition assessment in sport: Review and position statement on behalf of the ad hoc research working group on body composition health and performance, under the auspices of the I.O.C. Medical Commission. Sports Medicine, 42(3), 227-249. PubMed ID: 22303996 doi:10.2165/11597140-000000000-00000

Barlow, M.J., Oldroyd, B., Smith, D., Lees, M.J., Brightmore, A., Till, K., ... Hind, K. (2015). Precision error in dual-energy X-ray absorptiometry body composition measurements in elite male rugby league players. Journal of Clinical Densitometry, 18(4), 546-550. PubMed ID: 26072358 doi:10.1016/j.jocd.2015.04.008

Bilsborough, J.C., Greenway, K., Livingston, S., Cordy, J., \& Coutts, A.J. (2016). Changes in anthropometry, upper-body strength, and nutrient intake in professional Australian football players during a season. International Journal of Sports Physiology and Performance, 11(3), 290-300. PubMed ID: 26217046 doi:10.1123/ijspp.2014-0447

Bilsborough, J.C., Greenway, K., Opar, D., Livingstone, S., Cordy, J., \& Coutts, A.J. (2014). The accuracy and precision of DXA for assessing body composition in team sport athletes. Journal of Sports Sciences, 32(19), 1821-1828. PubMed ID: 24914773 doi:10.1080/02640414. 2014.926380

Binkley, T.L., Daughters, S.W., Weidauer, L.A., \& Vukovich, M.D. (2015). Changes in body composition in Division I football players over a competitive season and recovery in off-season. Journal of Strength \& Conditioning Research, 29(9), 2503-2512. PubMed ID: 26313574 doi:10.1519/JSC.0000000000000886

Bone, J.L., Ross, M.L., Tomcik, K.A., Jeacocke, N.A., Hopkins, W.G., \& Burke, L.M. (2017). Manipulation of muscle creatine and glycogen changes DXA estimates of body composition. Medicine \& Science in Sports \& Exercise, 49(5), 1029-1035. doi:10.1249/MSS.00000000 00001174

Cornish, B.H., Ward, L.C., Thomas, B.J., Jebb, S.A., \& Elia, M. (1996). Evaluation of multiple frequency bioelectrical impedance and ColeCole analysis for the assessment of body water volumes in healthy humans. European Journal of Clinical Nutrition, 50(3), 159-164. PubMed ID: 8654329

De Lorenzo, A., Andreoli, A., \& Candeloro, N. (1997). Within-subject variability in body composition using dual-energy X-ray absorptiometry. Clinical Physiology, 17(4), 383-388. PubMed ID: 19361149 doi:10.1046/j.1365-2281.1997.04242.x

Dempster, P., \& Aitkens, S. (1995). A new air displacement method for the determination of human body composition. Medicine \& Science in Sports \& Exercise, 27(12), 1692-1697. PubMed ID: 8614327 doi:10. 1249/00005768-199512000-00017

Evans, E.M., Rowe, D.A., Misic, M.M., Prior, B.M., \& Arngrímsson, S.Á. (2005). Skinfold prediction equation for athletes developed using a four-component model. Medicine \& Science in Sports \& Exercise, 37(11), 2006-2011.

Fields, D.A., Higgins, P., \& Hunter, G. (2004). Assessment of body composition by air-displacement plethysmography: Influence of body temperature and moisture. Dynamic Medicine, 3(1), 3. PubMed ID: 15059287 doi:10.1186/1476-5918-3-3

Fields, D.A., Hunter, G.R., \& Goran, M.I. (2000). Validation of the BOD POD with hydrostatic weighing: Influence of body clothing.
International Journal of Obesity, 24(2), 200-205. PubMed ID: 10702771 doi:10.1038/sj.ijo.0801113

Gabbett, T.J. (2009). Physiological and anthropometric correlates of tackling ability in rugby league players. Journal of Strength \& Conditioning Research, 23(2), 540-548. PubMed ID: 19197211 doi:10.1519/JSC.0b013e31818efe8b

Hangartner, T.N., Warner, S., Braillon, P., Jankowski, L., \& Shepherd, J. (2013). The official positions of the international society for clinical densitometry: Acquisition of dual-energy X-ray absorptiometry body composition and considerations regarding analysis and repeatability of measures. Journal of Clinical Densitometry, 16(4), 520-536. PubMed ID: 24183641 doi:10.1016/j.jocd.2013. 08.007

Harley, J.A., Hind, K., \& O’Hara, J.P. (2011). Three-compartment body composition changes in elite rugby league players during a super league season, measured by dual-energy X-ray absorptiometry. Journal of Strength \& Conditioning Research, 25(4), 1024-1029. PubMed ID: 20651606 doi:10.1519/JSC.0b013e3181cc21fb

Hind, K., Slater, G., Oldroyd, B., Lees, M., Thurlow, S., Barlow, M., \& Shepherd, J. (2018). Interpretation of dual-energy X-ray absorptiometry-derived body composition change in athletes: A review and recommendations for best practice. Journal of Clinical Densitometry, 21(3), 429-443. PubMed ID: 29754949 doi:10.1016/j.jocd.2018.01. 002

Hume, P., Kerr, D.A., \& Ackland, T.R. (Eds.). (2018). Best practice protocols for physique assessment in sport. Singapore: Springer.

Hume, P., \& Marfell-Jones, M. (2008). The importance of accurate site location for skinfold measurement. Journal of Sports Sciences, 26(12), 1333-1340. PubMed ID: 18821122 doi:10.1080/026404108 02165707

Kerr, A., Slater, G.J., Byrne, N. (2017). Impact of food and fluid intake on technical and biological measurement error in body composition assessment methods in athletes. British Journal of Nutrition, 117(4), 591-601. doi:10.1017/S0007114517000551

Kerr, A., Slater, G.J., \& Byrne, N.M. (2018). Influence of subject presentation on interpretation of body composition change after 6 months of self-selected training and diet in athletic males. European Journal of Applied Physiology, 118(6), 1273-1286.

Kerr, A., Slater, G.J., Byrne, N.M., \& Chaseling, J. (2015). Validation of bioelectrical impedance spectroscopy to measure total body water in resistance-trained males. International Journal of Sport Nutrition and Exercise Metabolism, 25(5), 494-503. PubMed ID: 26011918 doi:10. 1123/ijsnem.2014-0188

Kerr, A., Slater, G.J., Byrne, N.M., \& Nana, A. (2016). Reliability of 2 different positioning protocols for dual-energy X-ray absorptiometry measurement of body composition in healthy adults. Journal of Clinical Densitometry, 19(3), 282-289. PubMed ID: 26343822 doi:10.1016/j.jocd.2015.08.002

Kyle, U.G., Bosaeus, I., De Lorenzo, A.D., Deurenberg, P., Elia, M., Manuel Gómez, J., ... Pichard, C. (2004). Bioelectrical impedance analysis-Part II: Utilization in clinical practice. Clinical Nutrition, 23(6), 1430-1453. PubMed ID: 15556267 doi:10.1016/j.clnu.2004. 09.012

Lambrinoudaki, I., Georgiou, E., Douskas, G., Tsekes, G., Kyriakidis, M., \& Proukakis, C. (1998). Body composition assessment by dualenergy X-ray absorptiometry: Comparison of prone and supine measurements. Metabolism: Clinical and Experimental, 47(11), 1379-1382. doi:10.1016/S0026-0495(98)90309-2

Lees, M.J., Oldroyd, B., Jones, B., Brightmore, A., O’Hara, J.P., Barlow, M.J., ... Hind, K. (2017). Three-compartment body composition changes in professional rugby union players over one competitive season: A team and individualized approach. Journal of Clinical 
Densitometry, 20(1), 50-57. PubMed ID: 27161801 doi:10.1016/j. jocd.2016.04.010

Marfell-Jones, M.J., Stewart, A., \& de Ridder, J. (2012). International standards for anthropometric assessment. Wellington, New Zealand: International Society for the Advancement of Kinanthropometry.

McCrory, M.A., Molé, P.A., Gomez, T.D., Dewey, K.G., \& Bernauer, E.M. (1998). Body composition by air-displacement plethysmography by using predicted and measured thoracic gas volumes. Journal of Applied Physiology, 84(4), 1475-1479. PubMed ID: 9516218 doi:10.1152/jappl.1998.84.4.1475

Meyer, N.L., Sundgot-Borgen, J., Lohman, T.G., Ackland, T.R., Stewart, A.D., Maughan, R.J., . .. Müller, W. (2013). Body composition for health and performance: A survey of body composition assessment practice carried out by the Ad Hoc Research Working Group on Body Composition, Health and Performance under the auspices of the IOC Medical Commission. British Journal of Sports Medicine, 47(16), 1044-1053. PubMed ID: 24065075 doi:10.1136/bjsports-2013092561

Nana, A., Slater, G.J., Hopkins, W.G., \& Burke, L.M. (2012a). Effects of daily activities on dual-energy X-ray absorptiometry measurements of body composition in active people. Medicine \& Science in Sports \& Exercise, 44(1), 180-189. doi:10.1249/MSS.0b013e318228b60e

Nana, A., Slater, G.J., Hopkins, W.G., \& Burke, L.M. (2012b). Techniques for undertaking dual-energy X-ray absorptiometry whole-body scans to estimate body composition in tall and/or broad subjects. International Journal of Sport Nutrition and Exercise Metabolism, 22(5), 313-322. doi:10.1123/ijsnem.22.5.313

Nana, A., Slater, G.J., Stewart, A.D., \& Burke, L.M. (2015). Methodology review: Using dual-energy X-ray absorptiometry (DXA) for the assessment of body composition in athletes and active people. International Journal of Sport Nutrition and Exercise Metabolism, 25(2), 198-215. PubMed ID: 25029265 doi:10.1123/ijsnem.20130228

Norton, K., Whittingham, N., Carter, L., Kerr, D., Gore, C., \& MarfellJones, M. (1996). Measurement techniques in anthropometry. In N. Kevin (Ed.), Anthropometrica (1st ed., pp. 25-75). Kensington, Australia: University of New South Wales Press Ltd.

O’brien, C., Young, A., \& Sawka, M. (2002). Bioelectrical impedance to estimate changes in hydration status. International Journal of Sports Medicine, 23(5), 361-366.

Olds, T. (2001). The evolution of physique in male rugby union players in the twentieth century. Journal of Sports Sciences, 19(4), 253-262. PubMed ID: 11311023 doi:10.1080/026404101750158312

Pace, N., \& Rathbun, E.N. (1945). Studies on body composition. 3. The body water and chemically combined nitrogen content in relation to fat content. Journal of Biological Chemistry, 158, 685-691.

Rouillier, M.A., David-Riel, S., Brazeau, A.S., St-Pierre, D.H., \& Karelis, A.D. (2015). Effect of an acute high carbohydrate diet on body composition using DXA in young men. Annals of Nutrition and
Metabolism, 66(4), 233-236. PubMed ID: 26183608 doi:10.1159/ 000435840

Ruiz, L., Colley, J.R., \& Hamilton, P.J. (1971). Measurement of triceps skinfold thickness. An investigation of sources of variation. British Journal of Preventive and Social Medicine, 25(3), 165-167. PubMed ID: 5564960

Saunders, M.J., Blevins, J.E., \& Broeder, C.E. (1998). Effects of hydration changes on bioelectrical impedance in endurance trained individuals. Medicine \& Science in Sports \& Exercise, 30(6), 885-892. PubMed ID: 9624647

Siri, W.E. (1961). Body composition from fluid spaces and density: Analysis of methods. Nutrition, 9(5), 480-491.

Slater, G.J., Rice, A.J., Mujika, I., Hahn, A.G., Sharpe, K., \& Jenkins, D.G. (2005). Physique traits of lightweight rowers and their relationship to competitive success. British Journal of Sports Medicine, 39(10), 736741. PubMed ID: 16183770 doi:10.1136/bjsm.2004.015990

Smart, D.J., Hopkins, W.G., \& Gill, N.D. (2013). Differences and changes in the physical characteristics of professional and amateur rugby union players. Journal of Strength \& Conditioning Research, 27(11), 3033-3044. PubMed ID: 23603998 doi:10.1519/JSC. $0 \mathrm{~b} 013 \mathrm{e} 31828 \mathrm{c} 26 \mathrm{~d} 3$

Tegenkamp, M.H., Clark, R.R., Schoeller, D.A., \& Landry, G.L. (2011). Effects of covert subject actions on percent body fat by air-displacement plethsymography. Journal of Strength \& Conditioning Research, 25(7), 2010-2017. PubMed ID: 21499137 doi:10.1519/ JSC.0b013e3181e741b6

Toombs, R.J., Ducher, G., Shepherd, J.A., \& De Souza, M.J. (2011). The impact of recent technological advances on the trueness and precision of DXA to assess body composition. Obesity, 20(1), 30-39. PubMed ID: 21760631 doi:10.1038/oby.2011.211

Vescovi, J.D., Zimmerman, S.L., Miller, W.C., \& Fernhall, B. (2002). Effects of clothing on accuracy and reliability of air displacement plethysmography. Medicine \& Science in Sports \& Exercise, 34(2), 282-285. PubMed ID: 11828238 doi:10.1097/00005768-20020 2000-00016

Vitale, K.C., Owens, R., Hopkins, S.R., \& Malhotra, A. (2019). Sleep hygiene for optimizing recovery in athletes: Review and recommendations. International Journal of Sports Medicine, 40(8), 535-543. PubMed ID: 31288293 doi:10.1055/a-0905-3103

Ward, L.C., Isenring, E., Dyer, J.M., Kagawa, M., \& Essex, T. (2015). Resistivity coefficients for body composition analysis using bioimpedance spectroscopy: Effects of body dominance and mixture theory algorithm. Physiological Measurement, 36(7), 1529-1549. PubMed ID: 26034992 doi:10.1088/0967-3334/36/7/1529

Zemski, A.J., Hind, K., Keating, S.E., Broad, E.M., Marsh, D.J., \& Slater, G.J. (2019). Same-day vs consecutive-day precision error of dualenergy X-ray absorptiometry for interpreting body composition change in resistance-trained athletes. Journal of Clinical Densitometry, 22(1), 104-114. PubMed ID: 30454952 doi:10.1016/j.jocd.2018.10.005 